\title{
Diftong: a tool for validating big data workflows
}

\author{
Raya Rizk ${ }^{1 *} \mathbb{D}$, Steve McKeever ${ }^{1}$, Johan Petrini ${ }^{2}$ and Erik Zeitler ${ }^{2}$
}

\author{
*Correspondence: \\ raya.rizk@im.uu.se \\ ${ }^{1}$ Department of Informatics \\ and Media, Uppsala \\ University, Kyrkogådsgatan \\ 10, 75313 Uppsala, Sweden \\ Full list of author information \\ is available at the end of the \\ article
}

\begin{abstract}
Data validation is about verifying the correctness of data. When organisations update and refine their data transformations to meet evolving requirements, it is imperative to ensure that the new version of a workflow still produces the correct output. We motivate the need for workflows and describe the implementation of a validation tool called Diftong. This tool compares two tabular databases resulting from different versions of a workflow to detect and prevent potential unwanted alterations. Row-based and column-based statistics are used to quantify the results of the database comparison. Diftong was shown to provide accurate results in test scenarios, bringing benefits to companies that need to validate the outputs of their workflows. By automating this process, the risk of human error is also eliminated. Compared to the more labourintensive manual alternative, it has the added benefit of improved turnaround time for the validation process. Together this allows for a more agile way of updating data transformation workflows.
\end{abstract}

Keywords: Big data, Data testing, Data validation, Data quality, Big data validation process, Big data validation tool, Big data workflow

\section{Introduction}

Due to the rapid development of data there has been an exponential growth in the number of applications that use big data techniques [1]. Many applications and workflows that process data in diverse domains and transform them into business value have been developed. In order for those transformations to provide solutions for business problems in organisations, it is necessary to perform data analysis based on valid inputs and then ensure the correctness and accuracy of the outputs produced at later stages [2]. Thus, process validation is necessary to check the results with the expected behaviour [3].

The demand for implementing effective and efficient validation methods has increased in order to ensure data quality in all processing phases [4]. Data quality can be defined as the degree to which the state of data serves its purpose in a given context [5]. According to Experian Data Quality [6], 75\% of businesses are wasting an average of $14 \%$ of their revenue due to poor data quality. In addition, Gartner research indicates that the average annual impact of such low data quality on organisations is as high as $\$ 9.7$ million [7]. This can also be linked to IBM's estimation in 2016 [8], where they stated that the yearly cost of poor quality data in the US alone was $\$ 3.1$ trillion. This is likely to worsen with the increased amount and complexity of data. It 
is critical to maintain the quality as it is essential to derive value from data that can have a profound impact on companies decision-making processes [9].

However, the large volume and fast velocity in which heterogeneous data is being generated and processed makes measuring data quality difficult [2]. Most big data sets lack clear structure since the data are extracted from a diversity of data sources. This poses challenges on big data testing processes [10]. Furthermore, manual data validation is difficult and inefficient [3] as mentioned in the Harvard Business Review [11] where about $50 \%$ of knowledge workers' time is wasted trying to identify and correct errors. Thus, automated validation is required to detect the effect of every data transformation that has occurred. Numerous papers have been published concerning big data and their applications, but they seldom address the validation process [3] and how to assure data quality during different stages of a big data application [5]. Most of the current tools only provide the common basic data validation functions, such as checking null values and data types, ranges, and constraints [5]. Thus, a more detailed data validation process that examines all values in a data set and detects potential errors is still lacking.

Companies develop workflows (data transformations) to process data continuously. Such workflows define and manage a series of steps that process and transform data [12]. They then store the new results in databases to be used for planning and decision-making, or as inputs to other workflows. This process can be protracted and complex as it deals with a large amount of data with many integrations. Consequently, managing such workflows can be difficult [13] and deploying them without validation can lead to undesirable outputs [3].

Driven by evolving business needs and due to the rapid increase in the volume and variety of data, these workflows often need to be improved and updated. A workflow can be improved through the implementation of more efficient and optimised transformations. The updates can be related to the inclusion of new data models or meeting changing business requirements. In addition, any infrastructure upgrade can affect the results of a transformation even if no changes to the workflow were conducted.

It is imperative to implement a data validation tool that automatically compares the outputs of a pre-updated workflow and its post-update counterpart. This is accomplished by taking the same inputs and indicating whether the results of the new workflow match the previous one, or whether it produces incorrect results, as illustrated in Fig. 1. Laranjeiro et al. [14] present an approach to test data-centric services with poor quality data and no validation. Their study highlighted vulnerabilities arising from poor data at both the application level but also at the middleware level too.

Starting from the needs of Klarna Bank AB, a fintech company, which offers online consumer credit and payment solutions [15], this study provides a new solution that attempts to tackle some of the big data validation problems. Klarna extends soft credit to consumers mainly by providing payment products and flexible credit lines in their Checkout product, which is integrated with numerous on-line merchants. To make credit and fraud assessments for each request in real-time, it is mission critical to have adequate data continuously available. Numerous heavyweight transformations are executed on a regular basis to provide data for these risk and fraud assessments. It is important that these transformations are accurate, quick, and scalable. 


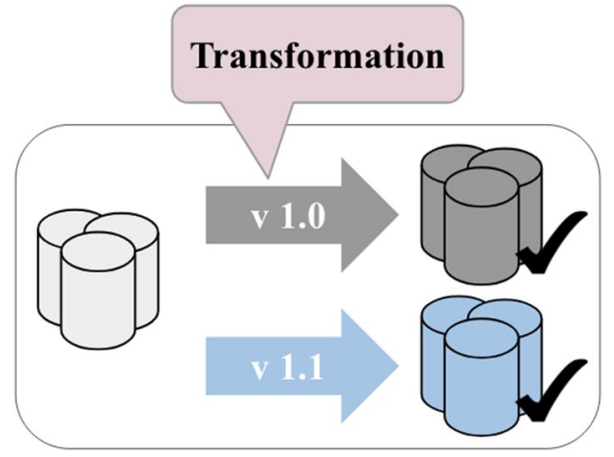

Fig. 1 Validating the outputs of two different versions of a workflow

The following two examples illustrate how Klarna leverages automatic comparison of databases in order to improve their company workflows:

Credit risk assessment capabilities

Fraud risk assessment capabilities
A workflow (w1) produces data for real-time credit risk assessment and decision making. To enhance data freshness $w 1$ was ported to a faster execution engine resulting in a new version of the workflow (w2). After running $w 1$ and $w 2$ side by side on the same input and doing automatic comparison of their respective result databases, some differences were identified. A root cause analysis was initiated with an effort to discard any potential non-deterministic behaviour within the workflow. This was accomplished by running $w 1$ in parallel with itself $\left(w 1_{\text {baseline }}\right)$. The same differences were noticed for $w 1$ and $w 1_{\text {baseline. }}$. Since the non-determinism was benign, it was concluded that enhancing data freshness, by moving to a faster execution engine, did not introduce any errors and the workflow was deployed in production.

A workflow $(w 1)$ produces data for real-time fraud risk assessment and decision making. To enhance data freshness $w 1$ was ported to a faster execution engine ( $w 2)$. An automatic comparison of $w 1$ and $w 2$ 's result databases showed some differences. Running $w 1$ against $w 1_{\text {baseline }}$ resulted in the same differences and identified non-determinism in the workflow. However, this time the differences were not benign but a consequence of semantic inconsistencies in the data. After updating the workflow to eliminate those inconsistencies, $w 1$ and $w 2$ were once again run in parallel. Their respective result databases were compared, found to be identical 
and the workflow was deployed in production. In this case, not only did the comparison give at hand that moving to a faster execution engine did not introduce any errors in the data, it also resulted in improved data quality.

In Klarna, automatic comparison of databases enables a more agile approach to workflow improvements and updates. More specifically, such comparisons:

1. Reduce the delay in improving workflow performance. This occurs through the rapid identification of any differences (rows and columns) in the result data produced by two versions of a workflow. This in turn makes it easier for the workflow owner to trust and verify the correctness of the improvement.

2. Improve the quality of the data due to easier identification of issues such as corrupt information and inconsistent semantics.

This paper presents a general solution for comparing any two databases that have the same schema. We will only focus on parts of the process validation stage of the overall big data testing process and not on the data staging or output validation that are explained later in the "Background" section.

The paper is structured as follows. The remainder of this section presents some related work in the field. In addition, it describes the background and covers the concept of big data, its testing and validation, in addition to the development frameworks that are used in this project. A detailed explanation of the proposed validation process and its implementation is listed in Section two. Section three introduces Diftong, our validation tool, and shows two test cases then analyses and discusses the results. Conclusions are presented in Section four.

\section{Related work}

Validation techniques have struggled to cope with the exploding size of data. Current research in big data quality considers the issue in broad terms, but there is little focus on how to effectively validate big data applications. Gao et al. [5] discuss big data validation and quality assurance by focusing on validation processes which includes data collection, cleaning, transformation, loading, analysis, and reporting. Their study presents a comparison of eight existing data validation tools concluding that all of them provide the basic validation criteria that are set in the paper such as checking data types, formats, ranges, logic, and null values, even if this comes with some limitations.

More detailed analysis was undertaken by the same authors a year later [9] where they conducted a case study and displayed the differences between the results that various data validation tools generate when validating the same set of data. It is worth noting that there is a lack of tools that generate descriptive statistics. Moreover, the study defines a quality checklist for big data including non-basic data validation types such as checking for duplication in data, inconsistency, and incompleteness. The authors reflect on the current data validation solutions and discuss the primary challenges and needs. In general, they stress that there is a gap in the available research in the area and that data quality issues are still open. Hence, it follows that further research and studies in 
this field should be conducted as only few published papers addresses big data validation methods.

Other studies focused on the general concept of data quality applied to big data. Firmani et al. [16] highlight that it could be challenging to define a unique concept of big data quality due to the fact that data quality is multidimensional and difficult to characterise even in the case of structured data. The authors attempt to define a method to apply the various notions of quality to specific types of big data. According to a classification proposed by the United Nations Economic Commission for Europe (UNECE), there are three main types of data sources in relation to big data: human-sourced information, process-mediated, and machine-generated sources. The paper describes a data model for each data source by quantifying data quality and its dimensions (accuracy, correctness, redundancy, readability, accessibility, consistency, usefulness, and trust) using metrics specific to the context and based on the characteristics of big data.

The overall aim of the study is to identify further research directions in the area of big data quality, and which was the base of another study conducted by Arolfo et al. [17] later on. In their paper [17], the authors also argue that data quality is dependent on the context and relative to the problem to be solved. In other words, different data quality dimensions and metrics can be considered for various applications areas. The paper defines how data quality dimensions can be used in a real-time scenario to address the quality of Twitter feeds. Thus, the study focuses on the human-sourced information type in reference to Firmani et al. [16], and only considers metrics for four dimensions related to this data source (readability, completeness, usefulness, and trust). On the other hand, our study can be considered as a part of the process-mediated type since it concerns banking, transactions, and e-commerce. Hence, the data is more structured than in the human-sourced generated type. As a result, the accuracy dimension (that is explained in "Background" section later) provides a valuable tool for improving the overall data quality in this type of applications as it deals with the closeness of values to a what is considered as the correct representation.

Both studies $[16,17]$ have emphasised that more research in the area is needed, as there has not been much work regarding data quality in the context of big data despite of the relevance of the topic.

There are some tools that attempt to validate big data. QuerySurge [18] is a tool that compares data that reside in two data stores (source and target). The input of this tool is two queries (QueryPairs) to be run against both source and target data stores, and the output is reflected in showing the difference in row numbers along with listing the values that differ in both stores. The tool comes with some limitations regarding a maximum row size of a result set and a maximum number of QueryPairs to compare in one run. QuerySurge is a closed source software that can be distributed under a licensing agreement.

Another example of a tool that attempts to calculate the delta between two values of large data sets is BigDiffy [19], an open source library for pairwise field-level statistical differences of data sets developed by Spotify. Delta is defined by Spotify as "a change of any changeable quantity" and the tool provides a record-oriented comparison that is undertaken on a columnar level based on unique keys. BigDiffy generates 
statistics and corresponds to the column validation step in Diftong, and which is a part of the whole validation process that is proposed in this paper.

\section{Background}

Relational Database Management Systems (RDBMS) have been the dominant model for more than 40 years. The relational model has a rigid schema with a clear structure [20]. Despite the robustness of RDBMS, it has limitations. It cannot handle the increasing size of data or process unstructured information [20].

Big data is more than just size, it is also varied and fast-growing. Hence it poses challenges to data management [21]. Non-relational databases do not use the RDBMS principles and do not store the data in tables [20]. The main characteristic of these databases is having a schema-free [22] format that results in unstructured data that the user has to interpret when retrieving it. The absence of structure, rules, and constraints makes it difficult to maintain clean and correct data without duplicates. It also affects the overall quality of information and introduces complexity in the testing and validation processes.

Testing software that uses big data techniques is significantly more complex than testing other more traditional data management applications. Simple test cases cannot be used for big data applications as processing the data requires that it be transferred and transformed at different points during each process of the application, costing time [1]. In order to test big data applications effectively, continuous validation throughout the transformation stages is advocated [23].

There are different types of tests that can be conducted to maintain the standard of data. Data quality includes various dimensions that should be measured such as data accuracy, correctness, redundancy, readability, accessibility, consistency, usefulness, and trust [24]. Data accuracy is usually measured by comparing the data in multiple data sources, as this quality factor refers to how close the results are to the values that are accepted as being true [5]. We mainly focus on this factor in the validation of data in our work.

The processing of big data, and thus its validation, can be divided into three different stages [25] as shown in Fig. 2:

1 Data staging: Loading data from various external sources. Validation includes verifying that the needed data were extracted and retrieved correctly, then uploaded into the system without any corruption.

2 Processing: In this step, it is required to validate the results of a parallelized job application and other similar big data application processes, while ensuring the accuracy and correctness of the data.

3 Output: Extracting the output results, and where validation includes checking whether the data have been loaded correctly into the target system for any further processing.

We used the Hadoop framework that supports parallel processing. It was designed to scale up to thousands of machines that offer local storage and computation. Hadoop achieves scalability and fault tolerance by distributing and replicating the data and 


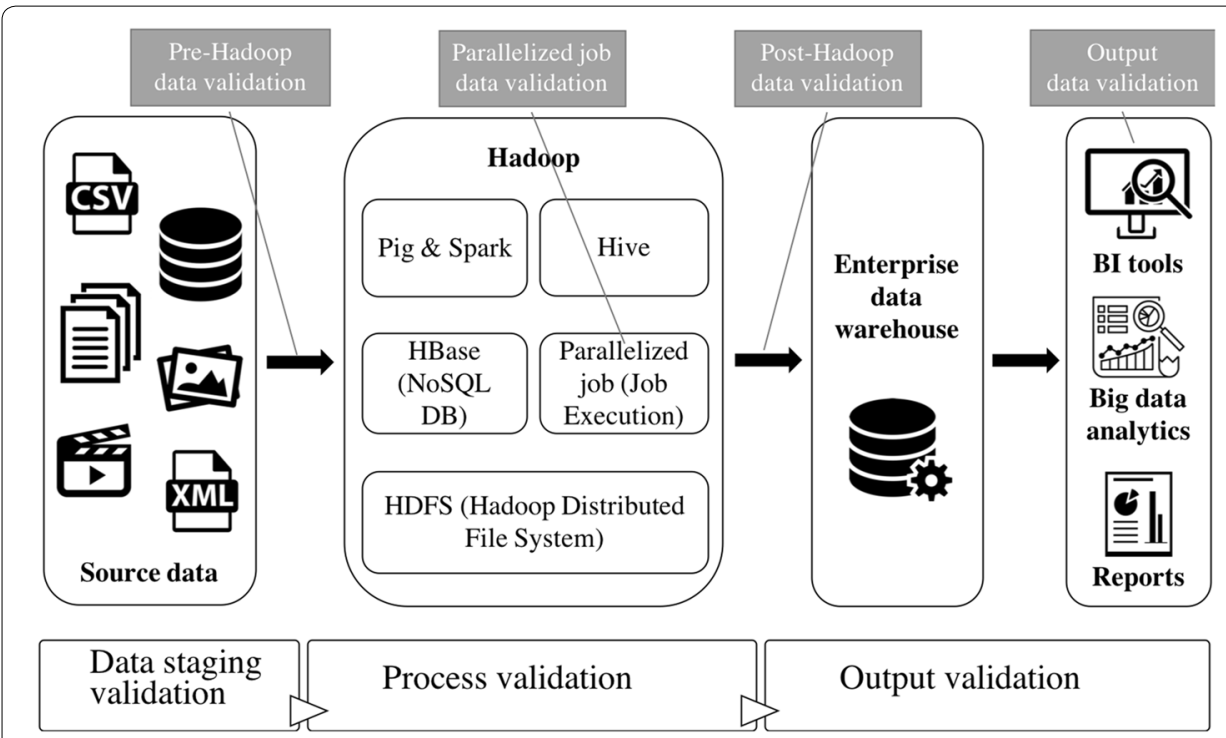

Fig. 2 Big data validation stages [26]

parallelizing the work across multiple nodes, effectively moving the computations closer to the data [27]. This helps with solving problems on large and complex data sets in a reliable manner, as it also provides automatic fault tolerance and recovery.

Apache Hive is a data warehouse built on top of Hadoop [28] to reduce the complexity of big data frameworks to gain easier access to the desired data. Due to the limited query capabilities of Hadoop and the complexity of the MapReduce framework, developers were required to write complex programs that might be hard to maintain and reuse even for simple analysis [29]. In contrast, Hive supports queries expressed in a declarative language similar to SQL-called HiveQL-and has its own Data Definition Language (DDL) and Data Manipulation Language (DML) commands. Those queries are compiled into MapReduce jobs that use the parallel processing in Hadoop [30]. Hive also provides a mechanism to project structure onto the large volume of data that reside in distributed storage. It facilitates the management of these data sets by executing queries using an SQL-like query language as it makes the data in Hadoop look like it is stored in tables that consist of a number of rows, and each row has a specified number of columns [29].

In order to make sense of the unstructured nature of big data, a certain level of metadata is to be expected. In Hive, this is commonly achieved through a meta-store service; it is a system catalog, created in a RDBMS, that contains within itself information and details about the objects definitions (schemas, tables, partitions, columns, etc.) [30]. By separating parsing instructions from the actual data, the meta-store acts as a form of index for otherwise impenetrable data files. In addition, meta-store deals with supplementary statistics concerning the data, providing utility in data exploration, query optimisation, and query compilation [29].

Conventionally, MapReduce has been the engine used to run Hive jobs in Hadoop. MapReduce has gradually been replaced by other more efficient execution engines, such as Tez and Spark. These execution engines are capable of executing more efficient Hive 
query execution plans, and are overall more resource efficient [30]. In particular, we have seen how one single Tez job can replace multiple MapReduce jobs in the applications.

A number of configuration variables in Hive are available to change the behaviour of the default installation settings. For instance, in order to get the number of rows in a table faster, Hive provides configurations to store the results of some queries-such as the COUNT statistics-in the meta-store [31]. This optimises the statistics collection as the values will be read from the meta-store directly instead of being calculated each time. Those variables can be configured using the SET command that works on a session level [32]. In addition, setting the "hive.stats.autogather" configuration to "true" enables automatic gathering and updating of statistics during operations, and "hive.compute. query.using.stats" answers queries like MIN, MAX, and COUNT solely using statistics stored in the meta-store.

\section{Methods and implementation}

This section describes the implementation of Diftong, which enables large scale validation of heavyweight data transformations. A small running example is used to illustrate the steps of the process.

To provide an overview of the changes, Diftong generates a statistical summary on both row and column levels for all tables. Furthermore, to help analysts and engineers perform root cause analysis of changes, Diftong also provides a detailed description of each difference in the form of a change capture. The validation is performed in three stages: Deduplication, Row validation, and Column Validation. Figure 3 displays a highlevel illustration of the validation process with a summary of the generated statistics.

Our running example consists of two databases "DB1" and "DB2" with identical schemas that are to be validated. Each database contains a table named "Users" with four columns (id, name, salary, and birthday) as displayed in Table 1, while Fig. 4 shows the data in the "Users" table present in both databases.

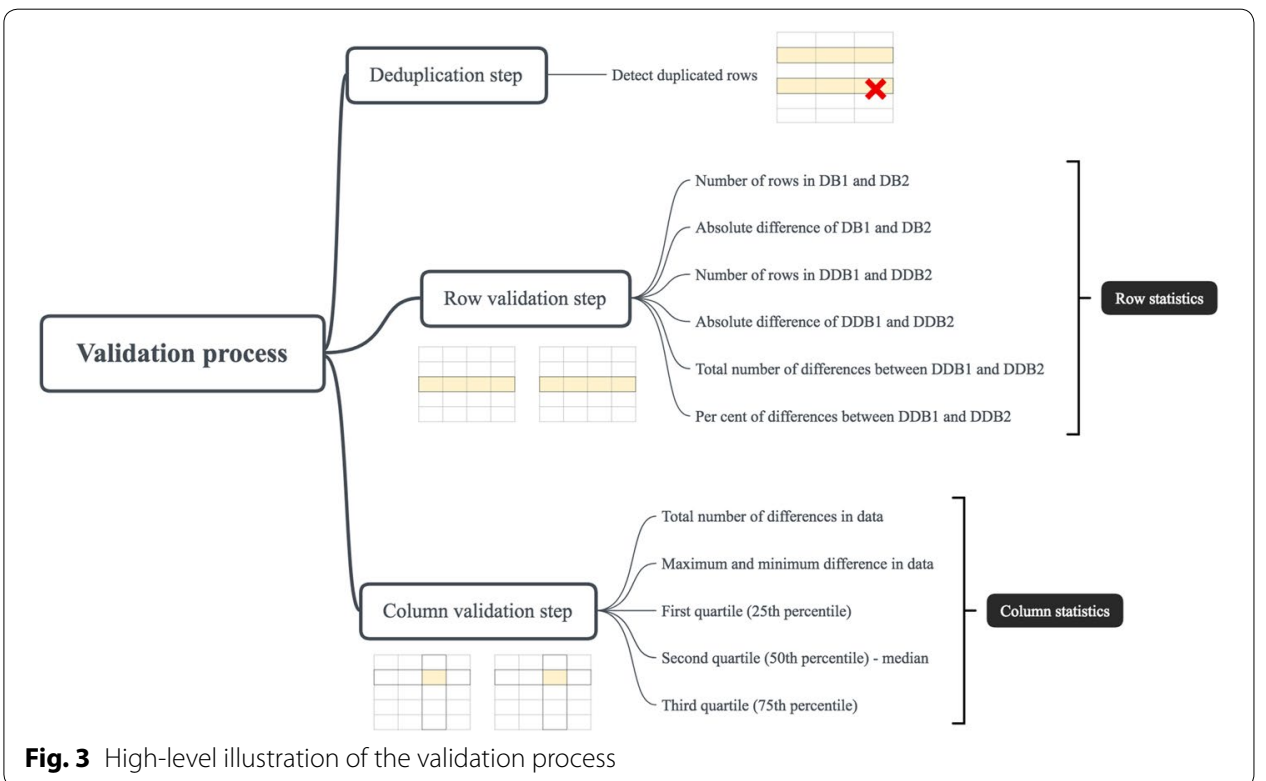


Table 1 Original databases schema_-“Users” table

\begin{tabular}{ll}
\hline Column name & Data type \\
\hline id & int \\
name & string \\
salary & int \\
birthday & timestamp \\
\hline
\end{tabular}

\begin{tabular}{|cccc|}
\hline \multicolumn{4}{c}{ DB1 } \\
id & name & salary & birthday \\
\hline 1 & John & 36000 & $1982-12-16$ 00:00:00 \\
\hline 2 & Mark & 31000 & $1987-06-2302: 00: 00$ \\
\hline 3 & Sofie & 25000 & $1990-08-07$ 08:00:00 \\
& & DB2 & birthday \\
\hline id & name & salary & $1982-12-16$ 00:00:00 \\
\hline 1 & John & 36000 & $1987-06-23$ 02:00:00 \\
\hline 2 & Mark & 31000 & $1987-06-23$ 02:00:00 \\
\hline 2 & Mark & 31000 & $1990-08-07$ 08:30:00 \\
\hline 3 & sofia & 25500 & $1992-09-22$ 10:15:00 \\
\hline 4 & Anna & 29000 & \\
\hline
\end{tabular}

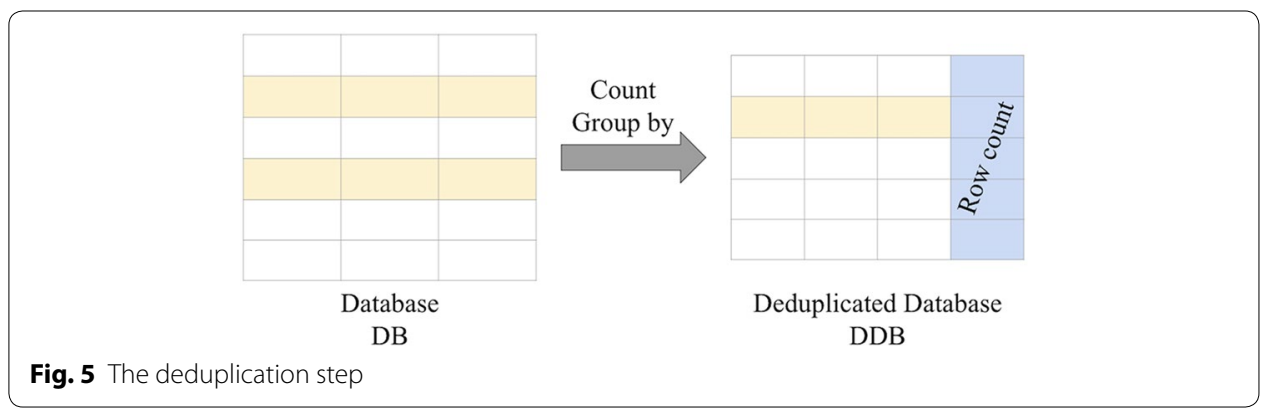

The validation stages are further detailed in the following sections.

\section{Deduplication}

As key constraints are not necessarily enforced in Hive, duplicate rows may be present. A duplication in data is where all values in one row match exactly all the values in another row. Each row in both databases is counted and the results are stored for use in subsequent stages of the validation process. Figure 5 illustrates the deduplication step.

Based on the data in the "Users" table that is shown in Fig. 4, all records appear once in each table except for the record with the id $=2$ in "DB2". This record has two identical rows where the values of all columns are equal. Hence, the COUNT for all the rows is 1 except for the record with the id = 2 in "DB2", which is 2 instead. 
Table 2 Deduplicated databases schema_-“sers" table

\begin{tabular}{ll}
\hline Column name & Data type \\
\hline Id & int \\
Name & string \\
Salary & int \\
Birthday & timestamp \\
Row_count & int
\end{tabular}

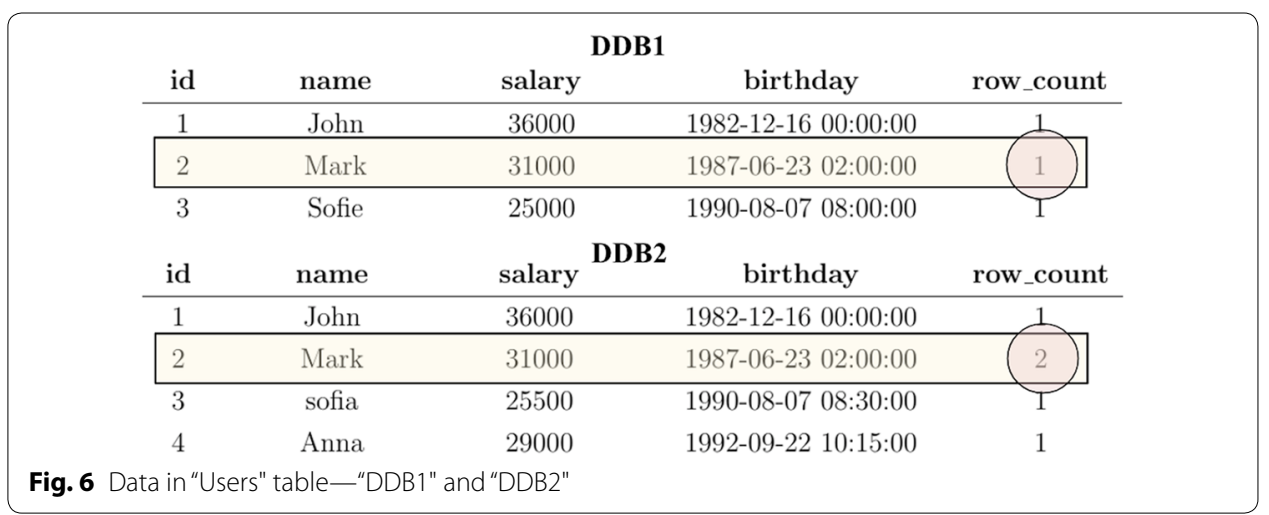

After the deduplication step, two new databases are created. Each database contains the same tables and data as the original databases before deduplication, but with one additional column "row_count" that shows the number of times each row appears in a table. Table 2 displays the schema of the databases "DDB1" and "DDB2"-deduplicated copies of "DB1" and "DB2" respectively-while Fig. 6 shows their data.

The results show that the different number of records for the user with id $=2$ is detected. With this count in place, it is easy to spot this discrepancy in the row validation stage described next.

The implementation steps for the deduplication stage are as follows. First, a deduplicated database instance is created by extracting all "CREATE TABLE" statements from DB1 and DB2. Each table is extended with an additional column called "row_count" as displayed in Script 1. By using COUNT and GROUP BY in this script, all duplicates in the original database are represented in the new deduplicated database using only one row with the extra column "row_count".

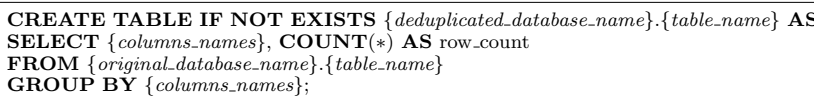

Script 1 Template for creating a table in the deduplicated database

\section{Row validation}

In order to get an overview of the total number of changes in each table, the differences between the rows of all tables in the two deduplicated databases are calculated. Consider the case of two databases "DB1" and "DB2", deduplicated respectively into "DDB1" and 


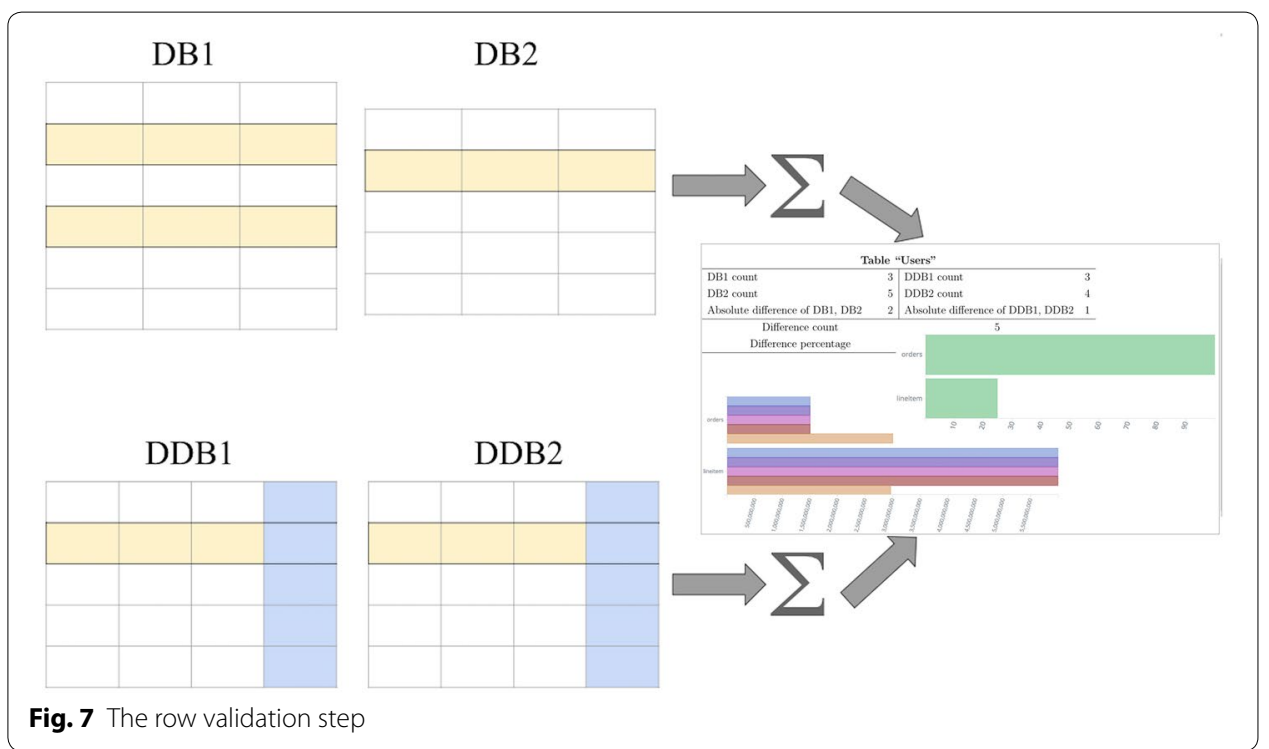

"DDB2". Figure 7 shows a simple representation of this step where the following statistics are generated:

Number of rows in $D B 1$

Number of rows in DB2

Absolute difference of $D B 1$ and $D B 2$

Number of rows in $D D B 1$

Number of rows in $D D B 2$

Absolute difference of $D D B 1$ and $D D B 2$

Total number of differences between $D D B 1$ and $D D B 2$

Per cent of differences between $D D B 1$ and $D D B 2$

To calculate the number of differences in the data values of each row (7) as a per cent (8), there is a need to detect which records in each table from "DDB1" have been changed in "DDB2" and what those changes are.

Figure 8 illustrates the differences in the values of all rows and columns in the "Users" table. It can be seen that five rows out of seven are different between "DDB1" and "DDB2", thus the difference is $71.4 \%$.

As a result, Table 3 displays the calculated row statistics for the "Users" table and depicts the number of changes on a row-based level with the difference as a per cent.

We describe how these statistics, from (1) to (8), are calculated as follows:

Row statistics (1), (2), (4), and (5): COUNT(*) in Hive is used to calculate the total number of rows in all tables of the databases "DB1", "DB2", "DDB1", and "DDB2". 


\begin{tabular}{|c|c|c|c|c|}
\hline \multicolumn{5}{|c|}{ DDB1 } \\
\hline id & name & salary & birthday & row_count \\
\hline 1 & John & 36000 & 1982-12-16 00:00:00 & 1 \\
\hline 2 & Mark & 31000 & 1987-06-23 02:00:00 & \\
\hline 3 & Sofie & 25000 & 1990-08-07 0\&:00:00 & 1 \\
\hline \multicolumn{5}{|c|}{ DDB2 } \\
\hline id & name & salary & birthday & row_count \\
\hline 1 & John & 36000 & 1982-12-16 00:00:00 & 1 \\
\hline 2 & Mark & 31000 & 1987-06-23 02:00:00 & 2 \\
\hline 3 & sofia & 25500 & 1990-08-07 0\$:30:00 & 1 \\
\hline 4 & Anna & 29000 & $1992-09-22$ 10:15:00 & 1 \\
\hline
\end{tabular}

Fig. 8 Row differences between "Users" table in "DDB1" and "DDB2"

Table 3 Row validation statistics_-“Users" table

\begin{tabular}{llll}
\hline DB1 count & 3 & DDB1 count & 3 \\
DB2 count & 5 & DDB2 count & 4 \\
Absolute difference of DB1 and DB2 & 2 & Absolute difference of DDB1 and DDB2 & 1 \\
Difference count & 5 & Difference in per cent & $71.4 \%$ \\
\hline
\end{tabular}

Row statistics (3) and (6): The absolute difference of the number of rows between ("DB1", "DB2") and ("DDB1", "DDB2") is calculated using the function ABS in Hive.

Row statistics (7): To calculate the total number of differences in the data of each table in "DDB1" and "DDB2", there is a need to compare the values in each row and detect any potential changes. Those differences and changes are referred to as the "delta" values. The differences are stored in row delta tables that help in generating the related statistics at a later step in this validation stage. The chosen method to store the delta tables is to create a new database that is used for both row and column validation stages.

One way to reflect changes in data is to represent each change as a record in the delta table. Each record contains the affected values with a validation status. The validation status can be either INSERT (when there is a new record in "DDB2" that does not exist in "DDB1") or DELETE (when a record exists in "DDB1" but not in "DDB2"). Note that the UPDATE status is actually a DELETE operation followed by an INSERT of the new values.

Table 4 displays an example of a possible representation of the differences that are shown in Fig. 8 for "Users" tables in both deduplicated databases, along with the related delta validation status. In other words, and based on the validation status of each record of the row delta calculation, applying the changes in sequence on the related table in the first deduplicated database will result in the data of the same table in the second one.

In order to implement the previous representation using Hive, delta values are calculated and stored in the newly created delta tables based on Script 2 . The row delta table contains all columns from both tables that are being compared along with the validation status that indicates what kind of change (INSERT or DELETE) has occurred on the data 
Table 4 Validation status for row delta table_-“Users" table

\begin{tabular}{llllll}
\hline Id & Name & Salary & Birthday & Row_count & Validation_status \\
\hline 2 & Mark & 31,000 & $1987-06-2302: 00: 00$ & 1 & DELETE \\
2 & Mark & 31,000 & $1987-06-2302: 00: 00$ & 2 & INSERT \\
3 & Sofie & 25,000 & $1990-08-0708: 00: 00$ & 1 & DELETE \\
3 & Sofia & 25,500 & $1990-08-0708: 30: 00$ & 1 & INSERT \\
4 & Anna & 29,000 & $1992-09-2210: 15: 00$ & 1 & INSERT \\
\hline
\end{tabular}

Table 5 Validation database schema_-“Users_row_delta" table

\begin{tabular}{ll}
\hline Column name & Data type \\
\hline Id1 & int \\
Name1 & string \\
Salary1 & int \\
Birthday1 & timestamp \\
Row_count1 & int \\
Id2 & int \\
Name2 & string \\
Salary2 & int \\
Birthday2 & timestamp \\
Row_count2 & int \\
Validation_status & string \\
\hline
\end{tabular}

in those tables. Table 5 shows the schema of the newly created validation database "ValidationDB" with the "Users_row_delta" table.

In Script 2, a full join between the tables in "DDB1" and "DDB2" is applied based on a unique value that identify each record, and the related validation status is then calculated. Lastly, the result is filtered to get the rows that are only represented in either table.

Note that since there are no primary keys to identify the rows in a big data set, a new user defined hash function, "multi_hash", was implemented to generate a unique identifier for each row based on the values in all its columns. The function "multi_hash" takes one input argument as an array of strings and concatenates its values taking into account the length of each string, then returns the SHA-512 hash for the concatenation of all string values in the input array. This is done to prevent any collision that might appear from moving text from one string to the next. Thus, moving any text will not produce collisions since the lengths would change. As an example, applying "multi_hash" function on the array of strings ['hello, 'world!'] (the concatenation is 'hello5world!6') results in a hex string that is different than the generated hash from the same function for the array ['hellow', 'orld!'], (the concatenation is 'hellow6orld!5') as the length of each word is taken into account. The hash value that was obtained from "multi_hash" is then used to compare and join similar records in those tables. 


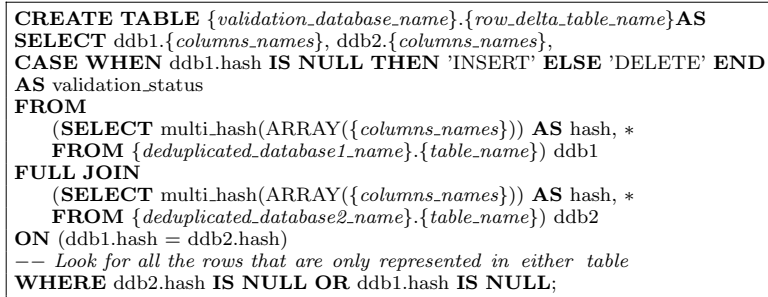

Script 2 Template for creating a row delta table in the validation database

As a result, Table 6 displays the items of data that have been changed with the related validation status column as the result of applying Script 2 on the "Users" table. A better representation of the data in "Users_row_delta" table is shown earlier in this section using Table 4.

After extracting the results from the row validation delta tables, the total number of differences in values of rows between "DDB1" and "DDB2" can be calculated based on counting the number of rows in those row delta tables in the validation database using COUNT $(*)$.

Row statistics (8): The per cent difference in each table is calculated by dividing the total number of differences that are calculated in (7) by the sum of the number of rows in both deduplicated databases tables, then multiplying the result by 100 and rounding it to one decimal place.

Running the "ANALYZE TABLE .. COMPUTE STATISTICS" query on all tables in "DDB1" and "DDB2" and on all row delta tables in the validation database helps in optimising the calculation of row statistics [31]. Note that tables in "DB1" and "DB2" were already analysed during the deduplication phase.

\section{Column validation}

Starting from the results of the row validation step, column based statistics are calculated for the tables that contain differences in order to get a deeper insight of the changes. It is important to first know how many changes have occurred and in what range. This can be achieved by calculating the total number of differences along with the minimum and maximum difference in the data. Calculating the average was also considered at the beginning but it did not give much added value to the results, so it was excluded from the generated statistics at a later stage.

Moreover, it is significant to measure the spread of the differences and get a deeper understanding of the data distribution. This can be obtained using the quartile statistics. By dividing the differences into four equal parts, the estimated quartiles and the median of the distribution can be calculated using 25th, 50th, and 75th percentiles of the data. Those percentiles reflect the first quartile (Q1-the middle number between the smallest number and the median), the second quartile (Q2-the median of the data), and the third quartile (Q3-the middle value between the median and the highest number in the data set) respectively [33]. Those measurements provide a better gauge of the overall data distribution as they ignore the values that are outside of the expected range, i.e. our measurements are not affected by outliers. 


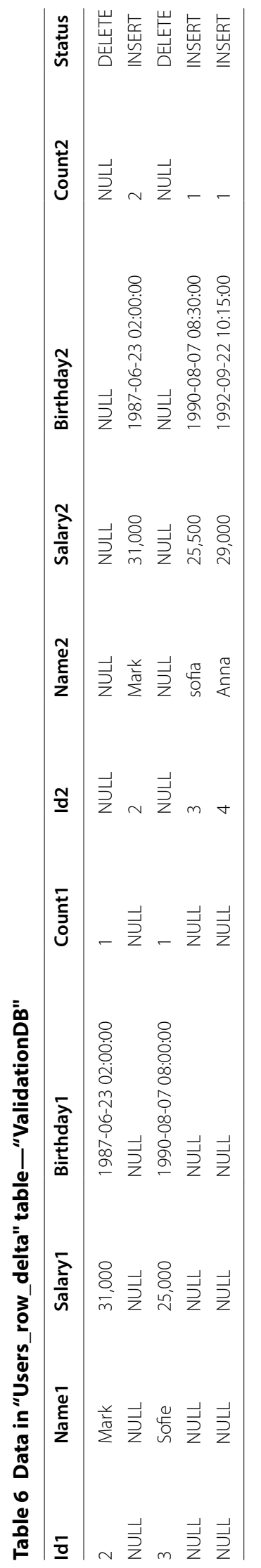


Consequently, the following statistics are generated for each column in the tables that are being validated:

Total number of differences in data

Maximum difference in data

Minimum difference in data

First quartile (25th percentile)

Second quartile (50th percentile) - median

Third quartile (75th percentile)

Consider the case of two databases "DDB1" and "DDB2", deduplicated copies of "DB1" and "DB2", in addition to a validation database "ValidationDB" that contains the row delta tables from the previous step. In order to generate the previous statistics, there is a need to compare and calculate the delta between each value inside the tables of "DDB1" and the related one in "DDB2". Figure 9 illustrates how each value is being compared in order to generate the needed statistics.

Figure 10 shows the differences in values of the columns in the "Users" table between "DDB1" and "DDB2". It can be seen that there is one difference in each of the columns. Note that any identical rows in both databases (such as the record with id $=1$ ), or rows that only exist in one of the databases (like the record with id $=4$ ) will not be a part of this validation stage.

As a result, Table 7 displays the calculated column statistics for the "Users" table. It shows the total number of differences in the data along with the related maximum and minimum delta values. In addition, it displays the three quartile values of each column. Notice that the numbers in all statistics match in this example due to having a single difference in each column.

The implementation of the column validation step starts with comparing the values in each column and calculating the delta between them. The differences are stored in new delta tables that help in generating the related statistics at a later step in this validation stage. Similar to the row validation stage, the column delta tables are stored in "ValidationDB".

For each record, the differences between all columns are calculated and stored in column delta tables that have the schema shown in Table 8.

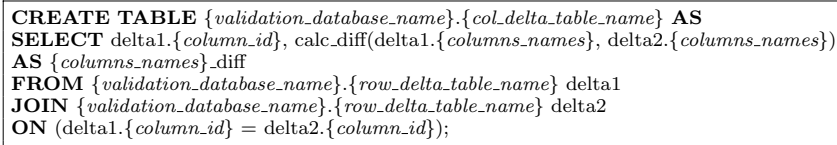

Script 3 Template for creating a column delta table in the validation database

Based on the data of row delta tables in "ValidationDB" (Table 6 is given as an example), column delta tables are generated using Script 3. First, a self join is applied on the row delta tables based on the id column of both "DDB1" and "DDB2" tables. Note that the id column should be provided by the user due to the lack of primary keys when 


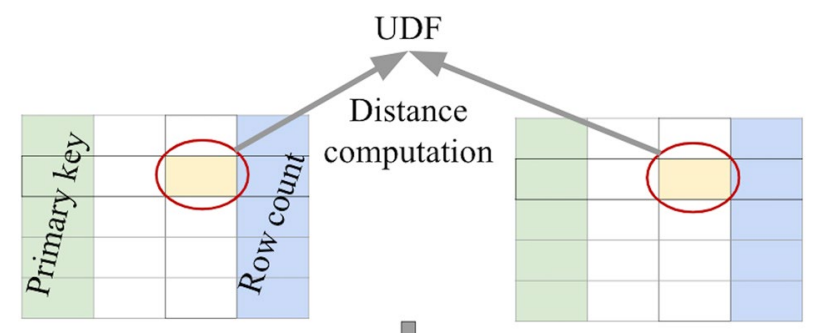

DDB2

Fig. 9 The column validation step

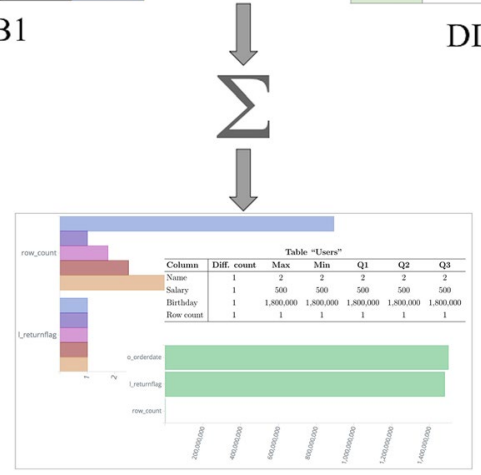

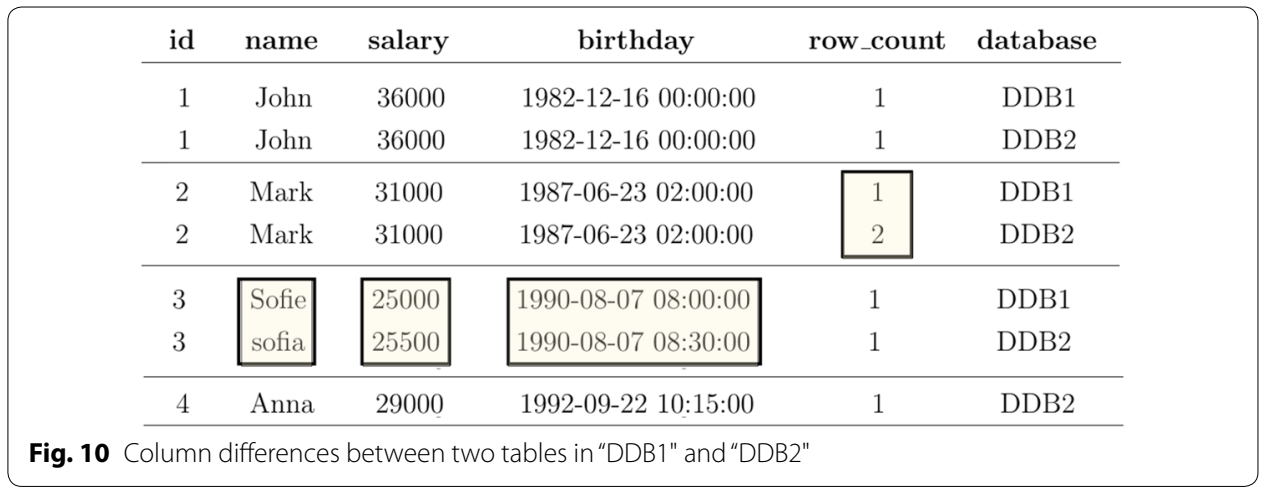

working with big data. Then, and for each column, the difference between all values of the tables in both deduplicated databases is calculated with the help of a user defined function, "calc_diff", that takes two values of the same type (integer, double, string, date, timestamp, or boolean) as parameters then calculates and returns the delta between them.

As a result, Table 9 displays the result of running Script 3 on "Users_row_delta" table. Note that NULL is stored if both values that are being compared are identical, i.e. the delta is equal to zero, or if both inputs are NULL.

Starting from the values in the column delta table, column statistics, from (9) to (14), are generated. A description of how they were generated is provided as follows:

Column statistics (9), (10), and (11): Calculating the count of changes along with the minimum and maximum differences for all columns can be costly. In order to optimise generating those statistics, and similar to the approach that is used in the previous validation stages, Hive configurations are set to obtain the values of MIN, MAX, and 
Table 7 Column validation statistics_“Users" table

\begin{tabular}{lllllll}
\hline Column & $\begin{array}{l}\text { Difference } \\
\text { count }\end{array}$ & Max & Min & Q1 & Q2 & Q3 \\
\hline Name & 1 & 2 & 2 & 2 & 2 & 2 \\
Salary & 1 & 500 & 500 & 500 & 500 & 500 \\
Birthday & 1 & $1,800,000$ & $1,800,000$ & $1,800,000$ & $1,800,000$ & $1,800,000$ \\
Row count & 1 & 1 & 1 & 1 & 1 & 1 \\
\hline
\end{tabular}

Table 8 Validation database schema_-“Users_col_delta" table

\begin{tabular}{lc}
\hline Column name & Data type \\
\hline id & int \\
name_diff & int \\
salary_diff & int \\
birthday_diff & int \\
count_diff & int \\
\hline
\end{tabular}

Table 9 Data in "Users_col_delta" table_“ValidationDB"

\begin{tabular}{lllll}
\hline id & name_diff & salary_diff & birthday_diff & count_diff \\
\hline 2 & NULL & NULL & NULL & 1 \\
3 & 2 & 500 & $1,800,000$ & NULL \\
\hline
\end{tabular}

COUNT using the statistics that are stored in the meta-store instead of calculating them each time.

Consequently, and in addition to analyzing the tables as in the row validation step, another script "ANALYZE TABLE .. COMPUTE STATISTICS FOR COLUMNS" [34] is executed to analyse column delta tables in "ValidationDB" and compute column-level statistics for all existing columns.

As for the next step, executing "DESCRIBE FORMATTED" script in HiveQL [34] on all the columns that hold information regarding the existing differences will generate the needed numbers to be used in the column statistics. The minimum and maximum values of differences are obtained along with the number of null values in each column, which reflect that no changes have been made to the data in the related fields of that column. As for the total number of changes, it is calculated by the subtraction of the number of null values from the total number of rows in the delta table. The latter is generated using COUNT (*).

Column statistics (12), (13), and (14): Hive provides a function called percentile that can be used to calculate the lowest and highest quartile of a data set along with the median value "percentile (BIGINT col, array (p1 [, p2] ... ))" [34]. This function calculates the specified percentiles for a data set, which is the list of differences for each column in this case. The provided percentile values are $(0.25,0.5,0.75)$ as $25 \%, 50 \%$, and 75\% reflect the values of Q1, Q2, and Q3. 
In this section we have shown how row-based and column-based statistics are generated by applying the aforementioned validation process. Row validation gives an overview of the total number of differences in each table with the per cent difference. Column validation, on the other hand, generates more detailed statistics such as the number of differences and the minimum and maximum difference in the data in each column. It also calculates the first quartile, the median, and the third quartile of the data. The analysis of those statistics is discussed in the next section.

\section{Results and discussion}

Diftong is our big data validation tool based on the validation process described previously. The tool has many features that create a solution for the validation of big data. It helps organisations to ensure the correctness of their transformations by detecting any changes in the generated data of different versions of the same workflow. In a more general context, the tool compares any two databases that have the same schemas and highlights the differences and similarities between them. Diftong could be included in a more robust information life cycle management framework as described in [35]. Similarly by ensuring data quality, big data analytics [36] can be applied with more trust [37].

Even though the tool was implemented using Hive and the Hadoop framework for the validation in a big data environment, the proposed validation process is generic and can easily be tailored to other table-based database management systems. Any key/value data set represented in the form of columns in a Hive table and queryable in HiveQL can be validated using the tool.

Diftong is easy-to-use and provides the ability to customise the validation process. This is achieved by giving the user the option to choose which steps to execute and which tables and/or columns to include or exclude from the validation.

\section{Test cases and analysis}

For the purpose of the analysis, two test cases were conducted on a test database with the size of $1 \mathrm{~TB}$. TPC-H is a decision support benchmark [38] that was used in the validation of the results of this study. A Hive testbench [39] that provides experiments with Apache Hive at any data scale was used to generate and populate a test database based on the TPC-H benchmark with a scale factor equal to 1000 (1TB). The TPC-H database consists of eight tables (Customer, LineItem, Nation, Orders, Part, PartSupp, Region, and Supplier) that contain several billions of records [38].

\section{First test case}

The Diftong tool was used to compare the previously stated test database-named "TPCH"-with an identical copy of it named "TPCH_copy". The data in both databases were first deduplicated, then the row validation stage was executed. As expected, no differences were found in any of the tables.

As a result, there is no need to run the column validation stage in this case. However, and in order to fulfil the purposes of this test case, the column validation step was executed anyway based on the results of the previous stages. Once again the tool behaved as expected by detecting that no differences were found and aborting the execution after 
displaying an information message to the user stating the reason behind not continuing with the execution of this stage.

\section{Second test case}

The following modifications were made to the data in the "TPCH_copy" database before executing the tool again:

- Table "LineItem":

New rows: One new row was added.

Duplication: Four rows were duplicated, the first was duplicated once, the second twice, the third three times, and the fourth ten times.

Column "L_returnflag": According to the specification of the TPC-H database [38], this column can contain one of the following values: "R", "A", or " $N$ ". All " $R$ " values were uncapitalised and replaced by " $\mathrm{r}$ ". The number of rows that contain this value is 1,480,675,200.

- Table "Orders":

Column "O_orderdate": All dates in this column were shifted 1 day forwards.

Row statistics Table 10 displays the new generated row statistics for the tables that contain differences. There is a 17-row difference ( 1 new row $+(1+2+3+10)$ duplicated rows $)$ in the "LineItem" table between the two databases before the deduplication step is applied. This number decreases to 1 (only the new row) where it reflects the delta between row numbers in both deduplicated databases.

As was explained in the previous section, the total number of differences can be calculated based on the number of changes that have occurred. In addition to the new row and the four duplications in rows, 1,480,675,200 values from the "TPCH" database were changed in "TPCH_copy" because of the modification that was undertaken in the column "L_returnflag". Thus, the total number of rows that are different in both databases and the per cent difference can be calculated as follows:

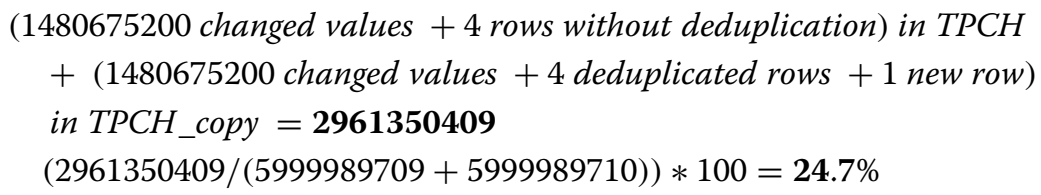

Similarly, it can be seen that there is $100 \%$ difference regarding the table "Orders" while no new rows were added and no rows were duplicated. In other words, all existing rows in the "ТРСH" database table are different from the related ones in the "TPCH_copy" database, and this is due to the change that was made on all dates in the "O_orderdate" column. As the results indicate, there is clearly a need to execute the column validation step in both tables "LineItem" and "Orders" in order to get more detailed insights regarding the changes that have occurred.

Figures 11 and 12 display the results of the row validation step in graphical interfaces as a part of the outputs of the Diftong tool. 
Table 10 Row validation statistics_-_Lineltem" and “Orders" tables

\begin{tabular}{lllllllll}
\hline Table & TPCH & TPCH_copy & $\boldsymbol{\Delta}$ & $\begin{array}{l}\text { Deduplicated } \\
\text { TPCH }\end{array}$ & $\begin{array}{l}\text { Deduplicated } \\
\text { TPCH_copy }\end{array}$ & $\boldsymbol{\Delta}$ & Difference count & $\%$ \\
\hline Lineltem & $5,999,989,709$ & $5,999,989,726$ & 17 & $5,999,989,709$ & $5,999,989,710$ & 1 & $2,961,350,409$ & 24.7 \\
Orders & $1,500,000,000$ & $1,500,000,000$ & 0 & $1,500,000,000$ & $1,500,000,000$ & 0 & $3,000,000,000$ & 100 \\
\hline
\end{tabular}

Column statistics The result of the row validation stage indicates which columns should be further investigated. In Table 11, the total number of differences that were caused by uncapitalising a letter in the "LineItem" table is reflected, with a minimum and maximum of 1 , which means that only one letter has changed. In addition, the four duplication operations are also listed with a range from 1 to 10 . The quartile values here give a good measure of the distribution of data, as it shows that smaller number of row duplications have occurred in general even if the maximum value is 10 , which is correct considering how the data was spread in this example (1,2, 3, and 10).

In some cases, the change is not necessarily unwanted. For example, the systematic change in the "O_orderdate" column in the "Orders" table shown in Table 11, may be a desired change. Desired changes may be excluded from the validation process by applying an exclude flag in Diftong. If this is the case, considering the differences in such columns might not give any added value, and thus they can be excluded from the validation process. Excluding the "O_orderdate" column in this example will make the per cent difference disappear.

Figures 13, 14, and 15 show the results of the column validation stage of the Diftong tool for both tables "LineItem" and "Orders".

\section{Performance measurements}

In order to estimate the effort for a validation using Diftong, execution time and CPUtime were measured for all queries in each test case (where databases of 1TB size were compared). Each experiment was repeated three times. Table 12 shows the average runtime and CPU-time for each test case, and Fig. 16 displays the chart related to the runtime measurements.

To put these $400 \mathrm{CPU}$-hours for a validation experiment in context, it is worth noting that counting all rows in both databases takes $\sim 3.5 \mathrm{CPU}$-hours. Thus, the computational cost of a validation using Diftong is comparable to the cost of running $\sim 100$ passes over the data.

It is notable that the measurement of the deduplication stage is similar in both test cases as the same database structures were used and only a few more rows were added to the data in the second case. However, the row validation step took longer compared to the first case since differences were detected and row statistics were calculated and generated. As for the column validation step, there was no time added for the first test case since no differences were found, while statistics were generated for both "LineItem" and "Orders" tables in the second case resulting in more time being added to the total number of milliseconds.

Moreover, by analysing and comparing the numbers in the two columns "First test case" and "Second test case", it can be noted that up to $80 \%$ of the time is devoted to the 

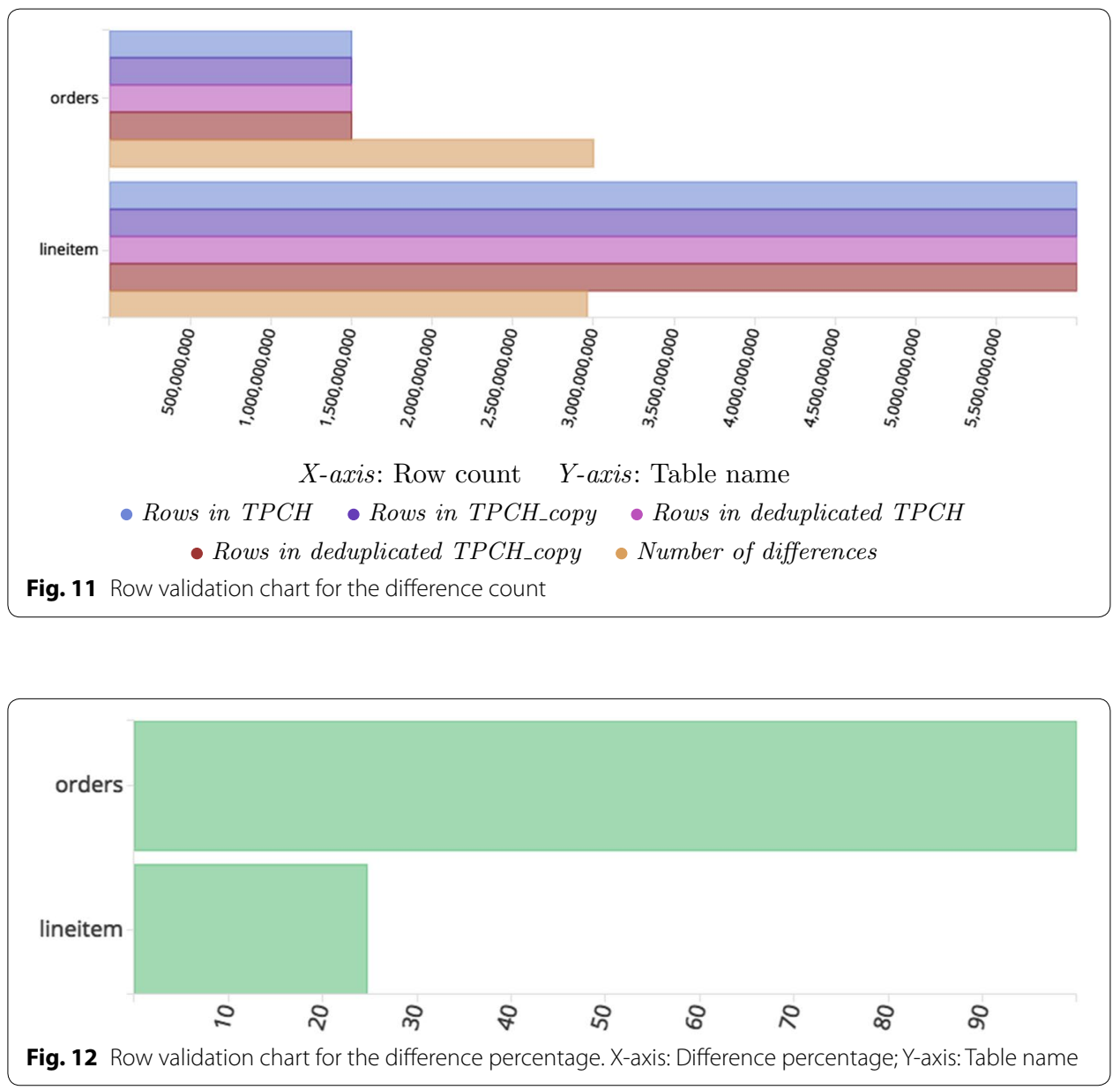

Table 11 Column validation statistics_-Lineltem" and “Orders" tables

\begin{tabular}{llllllll}
\hline Table & Column & Difference count & Max & Min & Q1 & Q2 & Q3 \\
\hline Lineltem & L_returnflag & $1,480,675,200$ & 1 & 1 & 1 & 1 & 1 \\
& row_count & 4 & 10 & 1 & 1.75 & 2.5 & 4.75 \\
\multirow{2}{*}{ Orders } & O_orderdate & $1,500,000,000$ & $90,000,000$ & $82,800,000$ & $86,400,000$ & $86,400,000$ & $86,400,000$ \\
\hline
\end{tabular}

deduplication stage, plus creating and populating row delta tables in the row validation stage in order to detect whether any differences have occurred or not. The cost of those two operations comes from the fact that deduplicating a table means that all rows in the table are grouped on all columns using the Hive GROUP BY operator, while row validating two tables (A and $\mathrm{B}$ ) means selecting all rows in $\mathrm{A}$ that are not in $\mathrm{B}$ and all rows in $B$ that are not in A; row validation was implemented using the Hive full outer join operator. For further analysis, it is interesting to investigate whether the deduplication step can be optimized. The work of Chu et al. should be considered [40].

The two test cases have demonstrated how the tool was able to detect every change that has occurred, providing results that help ensure the data accuracy. 


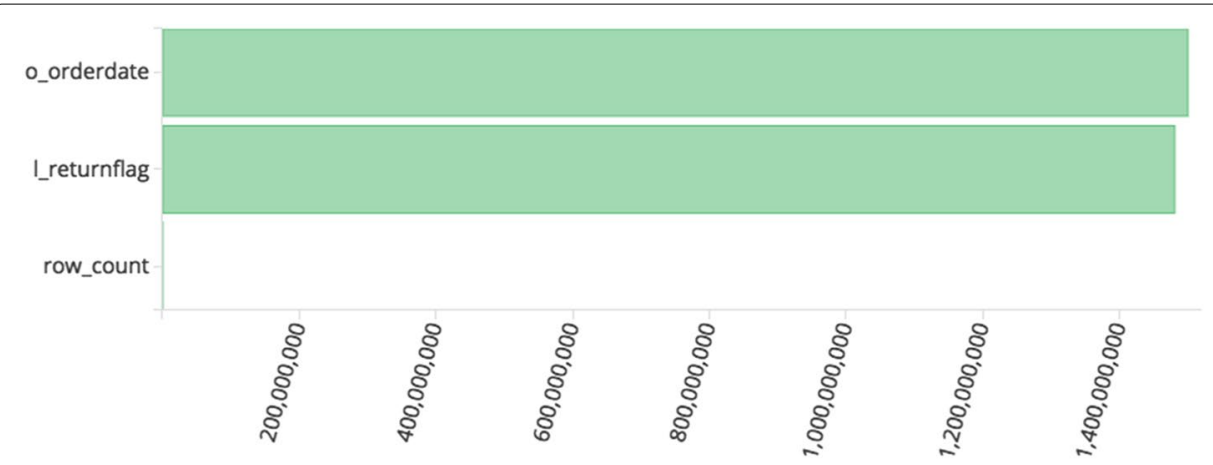

Fig. 13 Column validation chart for the difference count. X-axis: Difference count Y-axis: Column name
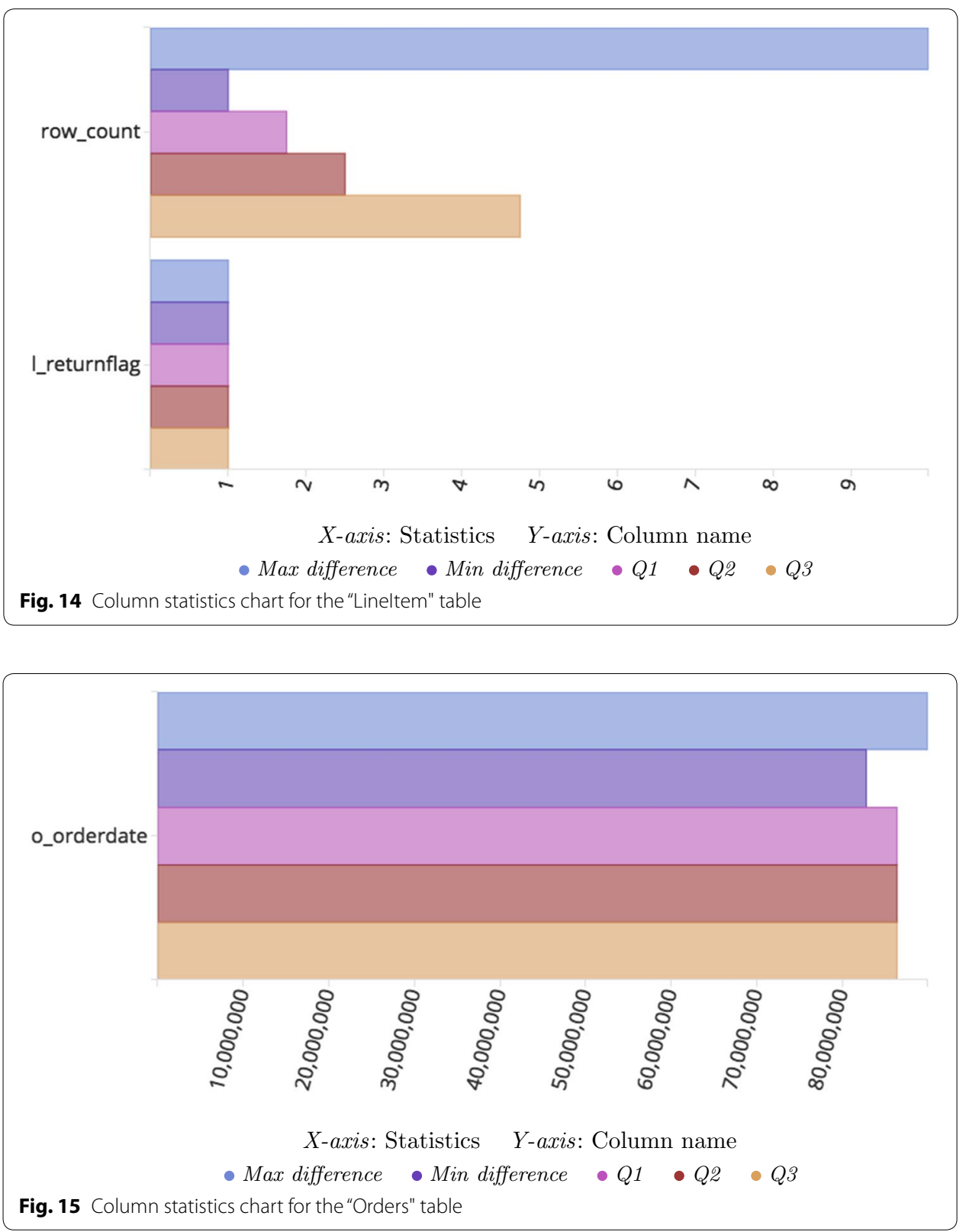
Table 12 Run-time and CPU-time for the two test cases

\begin{tabular}{lllllll}
\hline Validation stage & \multicolumn{2}{l}{ Run-time } & & & \multicolumn{2}{l}{ CPU-time } \\
& First test case & Second test case & & First test case & Second test case \\
\hline Deduplication & $3,627,636$ & $3,110,763$ & & $427,517,000$ & $465,107,430$ \\
Row validation & $2,162,731$ & $3,379,068$ & & $739,369,180$ & $835,724,920$ \\
Column validation & 0 & 599,418 & & 0 & $173,312,140$ \\
Total in milliseconds & $5,790,367$ & $7,089,249$ & & $1,166,886,180$ & $1,474,144,490$ \\
Total in hours & 1.6 & 2 & 324 & 409 \\
\hline
\end{tabular}

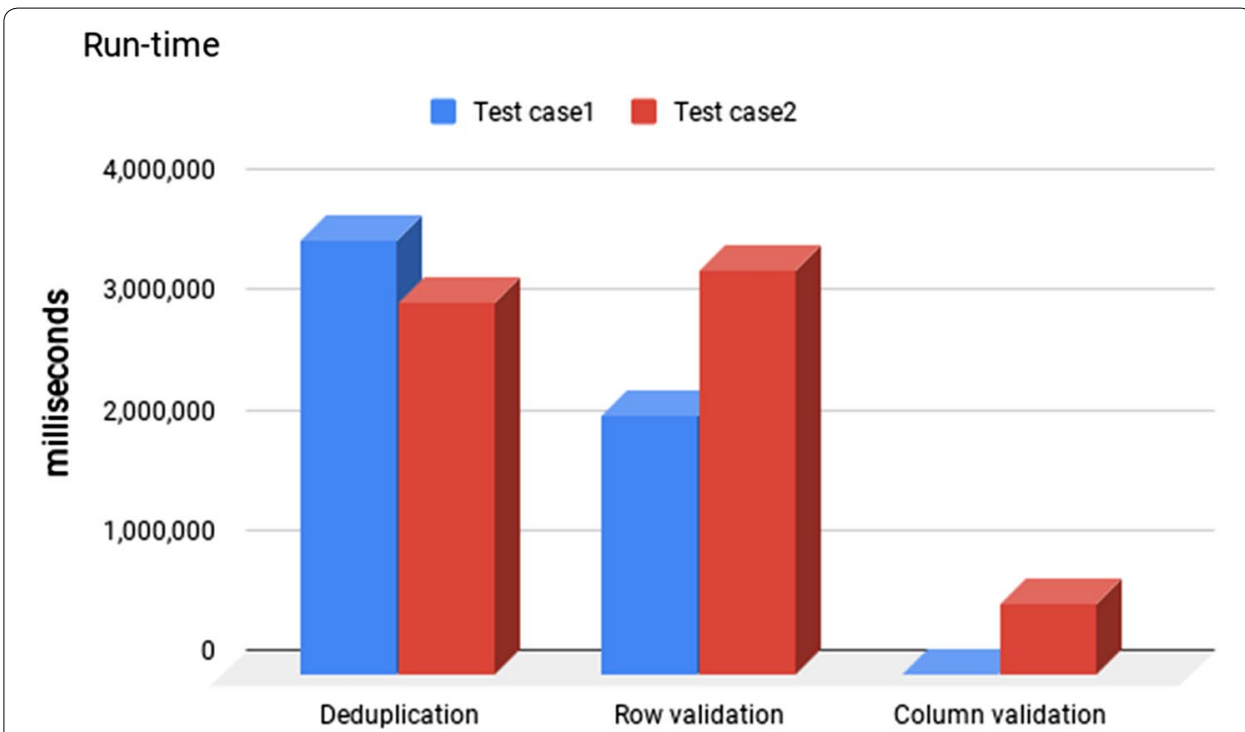

Fig. 16 Run-time chart for the two test cases. X-axis: Validation stage Y-axis: Run-time in milliseconds

\section{Conclusion}

Our work aimed to provide a general solution for scalable validation of data processing workflows. Diftong involves three stages: (i) deduplication, (ii) row validation, resulting in row statistics, and (iii) column validation resulting in column statistics. Row statistics give an overview of the data in all rows and identify the total number of discrepancies in each table with the aid of the per cent difference. Detecting the differences and similarities on a row-based level gives the user a clear idea of any changes that might have occurred. If this is the case, the user can ask for a deeper level of statistics based on each column in that table. Column statistics provide a more detailed view of the changes in data. The number of differences is calculated for each column along with the minimum and maximum difference in the data, which provides a further insight about the range of differences that have occurred. In addition, the quartile values (lower, middle, and upper) give more understanding of the distribution of data by measuring how differences are spread.

Diftong automatically computes overall statistics of the differences between two databases with identical schema. The automation of this process eliminates the timeconsuming manual labour and the risk of human error. Using Diftong in real use cases related to Klarna's core business has shown that the capability of identifying the 
differences between large scale databases helps organisations manage their data transformation workflows. In particular, Diftong has helped Klarna validate optimisations of workflows. It has also allowed the company to be more agile in their work with their data transformations, adding substantial value.

Using HiveQL, that provides standard SQL functionality, as the main development tool in this project delivers backwards compatibility and allows for interoperability between various database management systems. To validate data using Diftong, data have to be available in tabular form in Hive. Future work includes looking into adopting the tool to other storage engines and other query languages such as relational databases/ data warehouses, as well as key/value databases and graph databases.

Performance optimization of Diftong is a part of future work as well. For example, it is interesting to investigate whether the deduplication step can be optimized as it accounts for a majority of the execution time.

Finally, it should be considered whether Hive meta store could be extended to provide more statistics than the currently provided COUNT, MINIMUM and MAXIMUM values. Enriching Hive meta-store with additional descriptive statistics would facilitate the column validation stage of Diftong.

\section{Abbreviations \\ DDL: Data Definition Language; DML: Data Manipulation Language; RDBMS: Relational Database Management Sys- tems; UNECE: United Nations Economic Commission for Europe.}

\section{Acknowledgements}

The authors acknowledge the support from Uppsala University during the time of the study. The authors also acknowledge the help of the Klarna Bank AB and Klarna's employees at their Uppsala office for knowledge sharing and meaningful discussions. Jörgen Falk deserves a special mention for his continuous support in the implementation of Diftong.

\section{Authors' contributions}

$R R$, JP, and EZ designed the study and developed the methodology. RR wrote the manuscript, implemented the system, and performed the analysis under the supervision of JP. SM was the academic supervisor of the study, provided guidance with manuscript writing and was iteratively reviewing and revising it. All authors contributed to editing and evaluating the manuscript. All authors read and approved the final manuscript.

\section{Funding}

Not applicable.

Availability of data and materials

All data used during this study are publicly available and the source is included in the manuscript.

\section{Competing interests}

The authors declare that they have no competing interests.

Author details

${ }^{1}$ Department of Informatics and Media, Uppsala University, Kyrkogådsgatan 10, 75313 Uppsala, Sweden. ${ }^{2}$ Klarna Bank $A B$, Sveavägen 46, 11134 Stockholm, Sweden.

Received: 16 February 2019 Accepted: 6 May 2019

Published online: 18 May 2019

\section{References}

1. Li N, Escalona A, Guo Y, Offutt J. A scalable big data test framework. In: 2015 IEEE 8th international conference on software testing, verification and validation (ICST); 2015. p. 1-2. https://doi.org/10.1109/ICST.2015.7102619.

2. Cai L, Zhu Y. The challenges of data quality and data quality assessment in the big bata era. Data Sci J. 2015;. https:// doi.org/10.5334/dsj-2015-002.

3. Sadiq S, Orlowska M, Sadiq W, Foulger C. Data flow and validation in workflow modelling. In: Proceedings of the 15th Australasian database conference, Vol. 27. ADC '04, p. 207-214. Australian Computer Society, Inc., Darlinghurst, Australia, Australia; 2004. http://dl.acm.org/citation.cfm?id=1012294.1012317.

4. Taleb I, Dssouli R, Serhani MA. Big data pre-processing: a quality framework. In: 2015 IEEE international congress on big data; 2015. p. 191-198. https://doi.org/10.1109/BigDataCongress.2015.35. 
5. Gao J, Xie C, Tao C. Big data validation and quality assurance-issuses, challenges, and needs. In: 2016 IEEE symposium on service-oriented system engineering (SOSE); 2016. p. 433-441. https://doi.org/10.1109/SOSE.2016.63.

6. Experian data quality: the well-oiled data machine; 2014. https://www.edq.com/uk/blog/the-well-oiled-data-machi ne. Accessed 5 May 2018.

7. Moore S. How to create a business case for data quality improvement; 2017. https://www.gartner.com/smarterwit hgartner/how-to-create-a-business-case-for-data-quality-improvement. Accessed 12 May 2018.

8. IBM. Extracting business value from the 4 V's of big data; 2016. http://www.ibmbigdatahub.com/infographic/extra cting-business-value-4-vs-big-data. Accessed 12 May 2018.

9. Xie C, Gao J, Tao C. Big data validation case study. 2017 IEEE third international conference on big data computing service and applications (BigDataService). 2017; p. 281-286. https://doi.org/10.1109/bigdataservice.2017.44.

10. Garg N, Singla S, Jangra S. Challenges and techniques for testing of big data. Procedia Comput Sci. 2016;85:940-8. https://doi.org/10.1016/j.procs.2016.05.285 International Conference on Computational Modelling and Security (CMS 2016).

11. Redman TC. Data's credibility problem; 2013.https://enterprisersproject.com/sites/default/files/Data'sCredibilityPro blem.pdf.

12. Palazzo C, Mariello A, Fiore S, D'Anca A, Elia D, Williams DN, Aloisio G. A workflow-enabled big data analytics software stack for eScience. In: 2015 International conference on high performance computing simulation (HPCS); 2015. p. 545-552. https://doi.org/10.1109/HPCSim.2015.7237088.

13. Ordonez C, García-García J. Managing big data analytics workflows with a database system. In: 2016 16th IEEE/ACM international symposium on cluster, cloud and grid computing (CCGrid); 2016. p. 649-655. https://doi.org/10.1109/ CCGrid.2016.63.

14. Laranjeiro N, Soydemir SN, Ivaki N, Bernardino J. Testing data-centric services using poor quality data: from relational to NoSQL document databases. J Braz Comput Soc. 2017;23(1):14. https://doi.org/10.1186/s13173-017-0063-x.

15. Klarna: About us; 2018. https://www.klarna.com/se/om-oss. Accessed 29 Jan 2018.

16. Firmani D, Mecella M, Scannapieco M, Batini C. On the meaningfulness of "big data quality" (invited paper). Data Sci Eng. 2016;1(1):6-20. https://doi.org/10.1007/s41019-015-0004-7.

17. Arolfo F, Vaisman A. Data quality in a big data context. In: Benczúr A, Thalheim B, Horváth T, editors. Advances in databases and information systems. Cham: Springer International Publishing; 2018. p. 159-72. https://doi. org/10.1007/978-3-319-98398-1_11.

18. RTTS: QuerySurge; 2018. http://www.querysurge.com/solutions/testing-big-data. Accessed 04 June 2018.

19. Spotify: BigDiffy; 2018. https://github.com/spotify/ratatool/tree/master/ratatool-diffy. Accessed 04 June 2018.

20. Gyorödi C, Gyorödi R, Sotoc R. A comparative study of relational and non-relational database models in a webbased application. Int J Adv Comput Sci Appl. 2015;6(11):78-83. https://doi.org/10.14569/IJACSA.2015.061111.

21. Najafabadi MM, Villanustre F, Khoshgoftaar TM, Seliya N, Wald R, Muharemagic E. Deep learning applications and challenges in big data analytics. J Big Data. 2015;2(1):1. https://doi.org/10.1186/s40537-014-0007-7.

22. Mehmood NQ, Culmone R, Mostarda L. Modeling temporal aspects of sensor data for MongoDB NoSQL database. J Big Data. 2017;4(1):8. https://doi.org/10.1186/s40537-017-0068-5.

23. Geddam, S. Building a robust big data QA ecosystem to mitigate data integrity challenges; 2014 . https://www.cogni zant.com/whitepapers/building-a-robust-big-data-qa-ecosystem-to-mitigate-data-integrity-challenges-codex907. pdf. Accessed 12 May 2018.

24. Batini C, Monica S. Data and information quality: dimensions, principles and techniques. Switzerland: Springer; 2016 p. 21-51. https://doi.org/10.1007/978-3-319-24106-7.

25. Nagdive AS, Tugnayat DRM, Tembhurkar MP. Overview on performance testing approach in big data. International Journal of Advanced Research in Computer Science. 2014:5(8):165-169. https://www.researchgate.net/publicatio n/270338528_Overview_on_Performance_Testing_Approach_in_Big_Data. Accessed 12 May 2018.

26. Try QA: Big data testing; 2017 . http://tryqa.com/big-data-testing/. Accessed 13 Aug 2018.

27. Yassien AW, Desouky AF. RDBMS, NoSQL, Hadoop: a performance-based empirical analysis. In: Proceedings of the 2nd Africa and Middle East conference on software engineering. AMECSE '16, vol. 28-29, p. 52-59. ACM, New York, NY, USA; 2016. https://doi.org/10.1145/2944165.2944174.

28. Birjali M, Beni-Hssane A, Erritali M. Evaluation of high-level query languages based on MapReduce in big data. J Big Data. 2018;5(1):36. https://doi.org/10.1186/s40537-018-0146-3.

29. Thusoo A, Sarma JS, Jain N, Shao Z, Chakka P, Zhang N, Anthony S, Liu H, Murthy R. Hive-a petabyte scale data warehouse using Hadoop. In: Proceedings of the 26th international conference on data engineering, ICDE 2010, p. 996-1005. IEEE, Long Beach, California, USA; 2010. https://doi.org/10.1109/ICDE.2010.5447738.

30. Shaw S, Vermeulen AF, Gupta A, Kjerrumgaard D. Practical Hive: a guide to Hadoop's data warehouse system. 1st ed. Berkely, CA, USA: Apress; 2016.

31. Atlassian: Apache Hive configuration properties; 2018. https://cwiki.apache.org/confluence/display/Hive/Configurat ion+Properties. Accessed 15 Apr 2018.

32. Atlassian: Apache Hive admin manual configuration; 2017. https://cwiki.apache.org/confluence/display/Hive/ AdminManual+Configuration. Accessed 07 May 2018.

33. Žerovnik J, Poklukar DR. Elementary methods for computation of quartiles. Teach Stat. 2017;39(3):88-91. https://doi. org/10.1111/test.12133.

34. Atlassian: Apache Hive language manual; 2017. https://cwiki.apache.org/confluence/display/Hive/LanguageMa nual. Accessed 08 Apr 2018.

35. Moulos V, Chatzikyriakos G, Kassouras V, Doulamis A, Doulamis N, Leventakis G, Florakis T, Varvarigou T, Mitsokapas E, Kioumourtzis G, Klirodetis P, Psychas A, Marinakis A, Sfetsos T, Koniaris A, Liapis D, Gatzioura A. A robust information life cycle management framework for securing and governing critical infrastructure systems. Inventions. 2018; https ://doi.org/10.3390/inventions3040071.

36. Schneider J, Handali JP, vom Brocke J. Increasing trust in (big) data analytics. In: Matulevičius R, Dijkman R, editors. Advanced information systems engineering workshops. Cham: Springer; 2018. p. 70-84. 
37. Sänger J, Richthammer C, Hassan S, Pernul, G. Trust and big data: a roadmap for research. In: 2014 25th international workshop on database and expert systems applications; 2014. p. 278-282. https://doi.org/10.1109/DEXA.2014.63.

38. TPC: TPC Benchmark ${ }^{T M} H$ standard specification revision 2.17.3, San Francisco. Transaction processing performance council (TPC); 1993-2017. http://www.tpc.org/tpc_documents_current_versions/pdf/tpc-h_v2.17.3.pdf.

39. Hortonworks: Hive TestBench; 2018. https://github.com/hortonworks/hive-testbench. Accessed 16 May 2018.

40. Chu X, llyas IF, Koutris P. Distributed data deduplication. Proc VLDB Endowment. 2016;9(11):864-75. https://doi. org/10.14778/2983200.2983203

\section{Publisher's Note}

Springer Nature remains neutral with regard to jurisdictional claims in published maps and institutional affiliations.

Submit your manuscript to a SpringerOpen ${ }^{\circ}$ journal and benefit from:

- Convenient online submission

- Rigorous peer review

- Open access: articles freely available online

- High visibility within the field

- Retaining the copyright to your article

Submit your next manuscript at $>$ springeropen.com 